\title{
Vitamin C and COVID-19: should clinical trials be prioritised for low income settings and vitamin C-deficient populations?
}

\author{
Anitra C Carr and Sam Rowe
}

To THE EDITOR: Managing coronavirus disease 2019 (COVID-19) in resource-limited settings requires a number of considerations, including reducing or preventing requirements for respiratory support. ${ }^{1}$ Vitamin $C$ is a low cost, readily available therapy that the World Health Organization has highlighted for further research as an adjunctive intervention with biological plausibility for improving the clinical outcome of patients with COVID-19. ${ }^{2}$

Due to the higher requirements for the vitamin during severe infection, patients with pneumonia and sepsis have low vitamin $C$ levels and require intravenous administration of gram doses to restore its normal blood levels. ${ }^{3} \mathrm{~A}$ recent case series in Spain reported deficient levels of vitamin C $(<9 \mu \mathrm{mol} / \mathrm{L})$ in 17 of a cohort of 18 COVID-19 patients with acute respiratory distress syndrome. ${ }^{4}$ Another observational study in the United States indicated a mean vitamin C level of $22 \mu \mathrm{mol} / \mathrm{L}$ (standard deviation, $18 \mu \mathrm{mol} / \mathrm{L}$ ) in a cohort of 21 critically ill patients with COVID-19; the mean vitamin C level of the patients who died was half that of the survivors. ${ }^{5}$

Low vitamin C levels and high prevalence of hypovitaminosis $C$ and deficiency have been reported in low to middle income countries (LMICs), ${ }^{6}$ and many of the risk factors for COVID-19 overlap with risk factors for vitamin $C$ deficiency. ${ }^{7,8}$ People with chronically low vitamin $C$ levels are particularly susceptible to developing outright deficiency, and may be more likely to respond to vitamin C administration.

The findings of randomised controlled trials on vitamin $C$ can vary depending on the country where the studies were carried out, with non-US trials showing greater response to vitamin C intervention. ${ }^{9}$ A systematic search of global clinical trial registries (ClinicalTtrials.gov and the WHO International Clinical Trials Registry Platform) found only a handful of small trials of intravenous vitamin C monotherapy in COVID-19 patients in LMICs (ie, China and Iran, $n=50-140$ participants). ${ }^{10}$

The two largest vitamin $C$ intervention trials are being run out of Canada ( $n=800$; NCTO4401150) and Italy ( $n=500 ;$ NCT04323514). There are a few small intravenous vitamin C monotherapy and COVID-19 trials in the US registered on ClincialTrials.gov (eg, NCT04344184, $n=200$; and NCT04363216, $n=66$ ). Another trial at the Cleveland Clinic is assessing oral vitamin $C$ with and without zinc (NCT04342728, $n=520$ ). However, due to its pharmacokinetics, oral vitamin $\mathrm{C}$ may not be as efficacious as intravenous vitamin C. ${ }^{11}$

The baseline vitamin C levels of COVID-19 patients will likely affect their outcomes and their response to intervention. ${ }^{11,12}$ As such, further intravenous vitamin C trials for COVID-19 should be prioritised in LMICs and vitamin C-deficient populations. Furthermore, as a matter of urgency, intravenous vitamin $C$ arms should be introduced to current large adaptive trials (as has been done with REMAP-CAP, NCT02735707), particularly for trials running in countries with high COVID-19 caseloads. Finally, future meta-analyses of these trials could include subgroup analyses of studies comprising populations with low baseline vitamin $C$ levels.

Acknowledgements: Anitra Carr is the recipient of a Health Research Council of New Zealand Sir Charles Hercus Health Research Fellowship.

\section{Competing interests}

None declared.

\author{
Author details \\ Anitra C Carr ${ }^{1}$ \\ Sam Rowe 2,3 \\ 1 Nutrition in Medicine Research Group, Department of \\ Pathology and Biomedical Science, University of Otago, \\ Christchurch, New Zealand. \\ 2 Department of Clinical Sciences, Liverpool School of Tropical \\ Medicine, Liverpool, UK \\ 3 Barts NHS Hospital Trust, London, UK
}

Correspondence: anitra.carr@otago.ac.nz 


\section{LETTER}

\section{References}

1 Siow WT, Liew MF, Shrestha BR, et al. Managing COVID-19 in resource-limited settings: critical care considerations. Crit Care 2020; 24: 167.

2 World Health Organization. A coordinated global research roadmap: 2019 novel coronavirus. Geneva; 2020. https://www. who.int/blueprint/priority-diseases/key-action/Coronavirus_ Roadmap_V9.pdf (viewed Sept 2020).

3 Carr AC. Vitamin C in pneumonia and sepsis. In: Chen Q, Vissers $M$; editors. Vitamin C: new biochemical and functional insights. Oxidative stress and disease. Boca Raton, FL: CRC Press, Taylor and Francis; 2020. pp. 115-35.

4 Chiscano-Camón L, Ruiz-Rodriguez JC, Ruiz-Sanmartin A, et al. Vitamin C levels in patients with SARS-CoV-2-associated acute respiratory distress syndrome. Crit Care 2020; 24: 522.

5 Arvinte C, Singh M, Marik PE. Serum levels of vitamin C and vitamin $D$ in a cohort of critically ill COVID-19 patients of a North American community hospital intensive care unit in May 2020. A pilot study. Med Drug Discov 2020; doi: 10.1016/j. medidd.2020.100064 [Epub ahead of print].

6 Rowe S, Carr AC. Global vitamin C status and prevalence of deficiency: a cause for concern? Nutrients 2020; 12: 2008.

7 Carr AC, Rowe S. Factors affecting vitamin C status and prevalence of deficiency: a global health perspective. Nutrients 2020; 12: 1963.

8 Centers for Disease Control and Prevention. Assessing risk factors for severe COVID-19 illness. CDC, 2020. https://www. cdc.gov/coronavirus/2019-ncov/covid-data/investigationsdiscovery/assessing-risk-factors.html (viewed Sept 2020).

9 Hemilä H, Suonsyrjä T. Vitamin C for preventing atrial fibrillation in high risk patients: a systematic review and meta-analysis. BMC Cardiovasc Disord 2017; 17: 49.

10 Carr AC. A new clinical trial to test high dose vitamin $C$ in patients with COVID-19. Crit Care 2020; 24: 133.

11 Lykkesfeldt J. On the effect of vitamin $C$ intake on human health: how to (mis)interpret the clinical evidence. Redox Biol 2020; 34: 101532.

12 Carr AC. Micronutrient status of COVID-19 patients: a critical consideration. Crit Care 2020; 24: 349. 